\title{
The social process of escalation: a promising focus for crisis management research
}

\author{
Johan Bergström", Sidney Dekker ${ }^{2}$, James M Nyce ${ }^{3}$ and Isis Amer-Wåhlin ${ }^{4}$
}

\begin{abstract}
Background: This study identifies a promising, new focus for the crisis management research in the health care domain. After reviewing the literature on health care crisis management, there seems to be a knowledge-gap regarding organisational change and adaption, especially when health care situations goes from normal, to nonnormal, to pathological and further into a state of emergency or crisis.

Discussion: Based on studies of escalating situations in obstetric care it is suggested that two theoretical perspectives (contingency theory and the idea of failure as a result of incomplete interaction) tend to simplify the issue of escalation rather than attend to its complexities (including the various power relations among the stakeholders involved). However studying the process of escalation as inherently complex and social allows us to see the definition of a situation as normal or non-normal as an exercise of power in itself, rather than representing a putatively correct response to a particular emergency.

Implications: The concept of escalation, when treated this way, can help us further the analysis of clinical and institutional acts and competence. It can also turn our attention to some important elements in a class of social phenomenon, crises and emergencies, that so far have not received the attention they deserve. Focusing on organisational choreography, that interplay of potential factors such as power, professional identity, organisational accountability, and experience, is not only a promising focus for future naturalistic research but also for developing more pragmatic strategies that can enhance organisational coordination and response in complex events.
\end{abstract}

\section{Background}

This study will outline what has been identified as a promising focus of future research into health care crisis. Reviewing the research of health care crisis management, there seems to be a knowledge-gap concerning the process of organisational change and adaption, especially as a situation goes from normal, to non-normal and then further into a state of crisis. Following the identification of a knowledge-gap an outline of a research agenda will be presented, intended to support the study of the process of escalation in health care.

\section{Crisis}

There is a growing body of research that looks at crisis (or emergency) management in different health care

\footnotetext{
* Correspondence: Johan.Bergstrom@lucram.lu.se

1 Lund University Centre for Risk Assessment and Management, PO-Box 118SE-22100, Lund, Sweden

Full list of author information is available at the end of the article
}

settings. Defined by Runciman [1] a crisis is "the point in the course of a disease at which a decisive change occurs leading either to recovery or to death" (p. 156). The definition treats the notion of crisis in binary terms as a clear transition from a non-crisis state to a state of crisis. In the same way crisis management is defined as the efforts to recover from this loss of control [2]. In other words, crisis management is seen as the process of returning to the previous binary state or status. This process of recovery from a loss of control is typically dealt with by means of crisis management training. Team training concepts, with their origin in industries such as aviation [3], have been introduced into healthcare with the aim of enhancing non-technical skills such as leadership [4], situation awareness [5], communication, and decision making [6,7]. Team training typically is performed in simulated environments $[8,9]$ and researchers have put much effort into the development of these environments and methods to assess the effects of such training. However these assessment cycles tend
C Biomed Central

(C) 2012 Bergström et al.; licensee BioMed Central Ltd. This is an Open Access article distributed under the terms of the Creative Commons Attribution License (http://creativecommons.org/licenses/by/2.0), which permits unrestricted use, distribution, and reproduction in any medium, provided the original work is properly cited. 
to be tautological in that they only measure a certain set of crisis management skills like improved teamwork processes by ensuring certain "correct behaviours" of individuals [10]. There is also a debate whether enhancing these non-technical skills have the payoff those who advocate instruction and remediation of these kinds believe they actually have [11-14].

Previous research into health care crises acknowledge that the process(es) at the centre of any crisis (like the conditions and symptoms of the human whose life is in danger) can be complex, reflecting any number of sometimes even opposing goals as well as high uncertainty. Still the literature tends to treat organisation boundaries and the organisation itself as stable entities. However in any modern hospital different sub-organisations like the labour ward, the operation ward and the paediatrics ward are typically loosely coupled (with a high degree of self-governance and local adaption) to each other. In crisis situations however they can become tightly coupled and highly interdependent (e.g. when performing a Caesarean section). This can lead to new, emerging and inherently complex properties of the system as a whole ones that can be temporary or permanent or both [15]. ${ }^{\mathrm{a}}$ But when does this tight coupling occur? What characterises this coupling? Which actors become tightly coupled and why?

The questions asked offer a promising way forward for studying medical crises. Typical solutions to the problem of managing crises have taken the form of proposals to improve behaviour (as described above) and implementation of more rigid workplace routines and structures. However from a complexity perspective, such strategies seem to be problematic. Rather than focusing on individual behaviour, the complexity perspective turns instead to the notion of bounded (or local) rationality [16]. Because certain behaviour can seem irrational or even erroneous from one perspective (particularly in the aftermath of an incident) does not necessarily mean that it was irrational or erroneous at the time of the incident [17], making notions such as "violations" highly problematic [18]. When it comes to the implementation of protocols, rules and routines as solutions to issues of health care safety, the complexity perspective counsels caution and suggests there may be serious limits to epistemology that underlies them and consequently the kinds of solutions that so far emerged [19-22].

While these issues have not been extensively studied by medical researchers, scholars interested in naturalistic settings of joint cognitive systems have given them some attention [23]. Some examples of studies of health care complexity includes studies of safety under dynamic and changing conditions [24], the way in which technology, in emergencies, tightens interconnection across departments and specialities [25], how the implementation of new technology can affect the working environment [26], challenges differential understandings and representations of health information pose for end-users and patient safety [27-29], and the strategies used by healthcare staff to achieve resilience and avoid conflicts under high-pressure conditions [30,31]. Researchers of joint cognitive systems have began to develop theories that incorporate a complexity perspective into the understanding of medical failure and success [23]. Such ideas present an opportunity for the exploration and development of the notion of crisis-empirically as well as theoretically building further on these ideas, a new focus of analysis for health care crisis can emerge from looking at the notion of escalation.

\section{Escalation}

The process of escalation has previously been defined as a situation "where an initial irregularity develops into a continually deteriorating situation and starts affecting other areas in an accelerating tempo, with consequences that are difficult to overview and impossible to predict" [32] (p. 220). Woods and Patterson [33] outline that "the concept of escalation concerns a process - how situations move from canonical or textbook to non-routine to exceptional". This position acknowledges the need for studying the dynamic process preceding the definition of a state of crisis.

Furthermore Woods characterises escalating situations in the following ways:

- A cascade of effects occurs in the monitored process.

- Demands for cognitive activity increase as the problem cascades.

- Demands for coordination increase as the problem cascades.

- The cascade and escalation represent a dynamic process.

These are important characteristics that should encourage further analysis. The first point is that by linking cascade of effects to a monitored process risks uncoupling this process from its social context. The next two points follow from the first. It assumes that cognitive activity and socio-cultural coordination increase as a result of the cascading problem rather than treat both cognitive activity and these coordination efforts as an inherent part of the escalating process. This in turn can further divorce the escalation process from its social context and this can lead to arbitrary analytic divisions say between inside and outside the organisation.

Using examples from maternity wards, our focus so far has been on the escalating labour situations starting of as normal, but at some point changing to be defined otherwise followed by the involvement of more staff 
from different wards, professions and hierarchies. Such situations can deteriorate and at some point a decision to perform an emergency Caesarean section might be taken. This is followed by the treatment of not one but two patients (the child and the mother). In previous work we have, based on naturalistic studies, suggested that seemingly simple intervention decisions (such as when a midwife calls a physician for help) can be interpreted to be complex to the point that the model of strict adherence to best practice guidelines needs to be complemented with one of enhancing organisational diversity [19]. In this debate article, we will argue that not only standard practices for how to manage escalating situations needs to be critically reflected upon, but also current best practice ways of understanding crisis and emergencies in healthcare need to be rethought. Instead of viewing escalation as a process uncoupled from its social context, the present analysis suggests that it is necessary to outline a theory for escalation as a social process in which coordination and cognitive activity are highly interrelated. In this sense our model of escalation is not simply one of organisational escalation or clinical escalation, in fact we questions whether any analytical choice to separate the two can be fruitful. Rather we see both as highly interrelated and consequently an analysis of escalation cannot separate the two.

\section{Discussion}

Consistent with the principle of epistemological pluralism [34] this discussion will explore, using multiple theoretical perspectives, the problem of obstetrical interventions. Here these will be treated as partially overlapping and sometimes contradictory accounts generated around critical decision points. The intent here is to help develop a vocabulary and a scientific agenda that can help us better understand the social process of escalation and the role these event(s) plays in health care crisis.

\section{The contingency theory perspective}

Organisational contingency theory, which attempts to describe the best way to organise human work given a particular set of environmental conditions, assumes that quickly changing local conditions can best be handled by decentralised organisations. Having central decision makers involved in all organisational actions and responses can create unacceptable delays and information bottlenecks [35]. Rather, advocators of the theory emphasise that the more dynamic the environment, the more organic and immediate the organisational structure may become. Contingency theory typically invokes the example of military organisations, which are extremely hierarchical in peacetime with a heavy emphasis on planning, standardisation and top down protocol like drills and ceremony, with the discipline and power necessary to enforce all these aspects of life. On the battlefield however, these structures not only become less rigid and more flexible in response to fluid and haphazard situations but also flatten, with fewer explicit appeals made to rank. There can even be rank inversions where lowerranking personnel may be better positioned (and equipped with more local knowledge) to take appropriate action than higher-ranking officers [36].

These findings have been replicated in a number of studies of high-reliability organisations [37-39]. This suggests that the decentralisation of decision-making authority, especially in regard to safety issues, can permit rapid and appropriate responses to dangers by the people closest to the problems at hand [40]. Wildavksy called this "decentralised anticipation," and argues that micro-level entrepreneurial efforts can do more to improve safety than any centralised and restrictive topdown policies or structures [41]. High reliability theory also stresses the need for operational discretion at lower levels in the organisation. Even the lowest ranks on the deck of an aircraft carrier have the authority to suspend any take off or landing that might pose unnecessary risk [42]. As a result, high reliability has looked carefully at roles validation of minority opinion [38] and encouragement of dissent [43] can play in averting crisis, something echoed in the recommendation to treat nurses as a primary investment in patient safety [44].

When it comes to obstetric interventions, however, contingency theory does not seem to hold water. Rather than become a flatter, more organic organisation, obstetrics (and healthcare in general) seems to do something quite different in crisis and emergencies. This is to make more use, not less, of expertise embedded in hierarchy and structure $[45,46]$. For example, one of the most important intervention decisions for hospital midwives is whether to call a resident (physician) for assistance. ${ }^{\mathrm{b}}$ If this decision is made, the organisation seems to have added another layer in response to the escalating problem. Organisational changes occurring during health care escalations can be characterised as upward and outward "reach" to different levels and types of institutionalised hierarchy/competences. This seems to pick up tempo and pace if the situation deteriorates. During a crisis not only the charge of the patient is handed over to the physician, but also the control of the ward and the work carried out there at least in the "intermediate model" of labour care we studied. Midwifes essentially "step back" from the responsibility of the judgment characteristic of all aspects of normality [47]. Intervention decisions, in the terms of actively delivering the baby, and thus handling the crisis, then formally belong to the physician with the midwifes no longer seeing themselves 
as responsible, especially in any bureaucratic sense of the term, for whatever happens next [19].

This, from the point of view of contingency theory, seems counter intuitive. To understand situations like this we require new models and perspectives. In particular, what we do not understand very well is why hierarchy and structure (often invoked as lay and even "scientific" reasons for failure in crises and emergencies) sometimes function as very effective resources in healthcare crises. The literature studying healthcare professionals has largely focused on the relationship between novices and experts [48]. Some literature is also available on the models of competence and hierarchy that inform the health care system [49]. However, how expertise and authority "shift" during crisis is not something that the literature so far has not focused on.

\section{The explanatory model of incomplete interaction}

The addition of further layers of organisation in escalating emergencies generates what the healthcare literature typically characterises as "communication problems". The result is problems arising in emergencies and escalations are often treated as (or reduced to) a failure of communication [50,51]. To what extent this is an appropriation of a common sense discourse, one that equates communication disorders with institutional escalations or crises, it is too early to say. But note that what is at work here is a common fallacy derived from folk sociology: Namely if the individual or individuals act "correctly" the same will be true for the social institutions they work within.

This kind of thinking Durkheim years ago tried to correct still seems to underlie much of the literature on healthcare emergencies. Communication problems are typically the targets for safety intervention in healthcare [7-9,52] and it is difficult to know, given the present state of research, how positively these interventions have impacted. However to reduce problems of organisational choreography in an escalating crisis to simply a communication problem tends to "flatten out" perhaps prematurely the explanation curve. Other factors that might be at work here include institutionally mediated historical and temporal dimension (tempo) of these incidents. When factors like these are not acknowledged, there is a risk that other significant issues such as power and hierarchy are muted [53]. Unfortunately, in safety and organisational studies, the analysis and "solutions" that are often proposed to handle clinical error tend to finesse issues of this kind. What actually occurs is that these aspects (and the kinds of negotiated practices we have been discussing here) essentially "fall out" of most analyses of health care safety.

To explain escalation in terms of incomplete interactions between actors creates several analytic problems and resolutions. The first is the reduction of complex event chains and choreographies ${ }^{c}-$ ones that are seldom successfully handled by any linear model - to some kind of social atomism (or psychologism) in which a single actor is thought be to (and is often held) accountable for that particular crisis $[54,55]$. As well, the literature on healthcare and safety tends to valorise individual performance so both a "hero" is praised for his/her success, and/or individual actors are singled out for blame $[56,57]$. In fact, in most explanations of crises situation, there is a tendency not to "go above" the shop floor. This too reinforces the tendency in the literature, to provide to accounts of individual competence and failure. This of course says much about the value Westerners place on the individual and on the individual competence and autonomy. However this can mask the importance event chains and clinical chorography can have for healthcare institutions and clinical effectiveness.

\section{Escalation as a social process}

Treating escalation as a process decoupled from the organisation, and one that can be managed by the best practice routines (such as calling for assistance at the point where the situation is no longer being normal), holds the risk of underestimating "the social construction of the context that both legitimates a particular form of action and constitutes the world in the progress ... [so] we might begin to consider not what is the situation, but how it is situated" [58] (p. 1471). A simple example of how the process of situating constructs the obstetric situation could make use of the concept of active management of labour [59]. Here, midwifes are instructed not to accept patients to the delivery unit unless they are in active labour. The reasons for this are related to the conviction that labour is not a process that benefits from hospitalisation before labour has entered its "active phase". Accepting patients "too" early in the natural process of labour can well medicalise problems a delivery unit cannot handle typically well. This can lead to a situation where a normal birth is defined as a nonnormal labour (a definition that influences the social construction of escalating obstetrical situations) and the response is to medically induce labour. This can introduce considerable risk compared to the natural onset of labour. This construction and definition of context can also be a means to negotiate power (adding or not adding another layer of the hierarchy) and, every time it is applied, it also reinforces the medical hierarchy model.

In the healthcare literature, the shift of responsibility as a situation deteriorates could be written off as just a shift of responsibility required due to an increasingly more complex situation, to an actor with more knowledge (as the hierarchy-competence model does). However, when looked at closely, this explanation seems too facile. In fact almost all midwifes are well aware that 
they may often be calling someone with less experience and competence than themselves to handle crises or emergencies because in most deliveries one turns first a junior physician [19].

What these calls for an obstetrician do is in effect to mark an organisational transition, i.e., a shift from a normal situation to a crisis. In Foucault's terms, these calls enable midwives to renegotiate "the order of things" $[60,61]$, and this occurs through their redefinition (their release of control and authority) in a specific institutional "activity". Midwifes can wield considerable power in the delivery room because they can construct and define situations there as normal or not. For a midwife virtually any kind of ward event can become evidence for one definition or another regarding the ward's and patients' status. Any number and variety of factors like the emergency staff on duty, number of patients at the ward, the length of pregnancy, or time of the day can be cited by midwives as evidence that a situation is normal or not.

When viewed this way, an organisation's shift from normal state to crisis cannot simply be seen as a response to a deteriorating process. The example of the midwives here shows the need to see escalation as a social process - one constructed by those who can alter or improvise on on-going habitual practice sometimes even to the extent that it can throw normal practice into question. There is seemingly a paradox at work here. In healthcare crises, hierarchy, i.e., power can be invoked to redefine the situation so that alternatives to local practice emerge. At the same time, the redefinition of local practice can also confirm the logic and rationality of normal practice and hierarchy. It is this simultaneous, challenging, extending and re-legitimatising of traditional practice and hierarchy that occurs in almost any crisis. This helps explain why these events can be so refractory to most forms of social analysis. In other words, the structural and social processes at work in any escalation can be difficult to capture or analyse, especially given the standard models of health care work and health care organisations. Further, the construction of the contingencies (emphasised by the actors themselves) can mask the role that power and hierarchy play in the process. However, when we shift focus and view escalation as an inherently social process, the construction of events there can be seen, among other things, as an exercise of power - one that occurs in and as response to a complex social world.

Again, the kinds of organisational changes that can occur during an escalating situation in delivery units do not follow the patterns predicted by classical contingency theory. In health care for example, the response seems to be to further rely on (and build) hierarchical structures. However, analysing event chains, and the way these midwives first problematise and then legitimatise the handover of responsibility in a crisis can lead us closer to understanding how healthcare emergencies and crises get defined as such. In fact, it may be useful to trace out how hierarchical structures and local practice and resources mutually become redefined and extended during escalation process that precedes any crisis. This could have potentially more yield than for example continuing to focus on the role communication plays in crisis situations.

\section{Implications}

Taking account of the social processes of escalation offers a new promising focus for healthcare research one that has epistemological as well as practical/pragmatic implications. It is suggested that organisational choreography, involving an interplay of potential factors such as power, professional identity, organisational accountability, and experience, become a new focus for naturalistic action research [62]. Rather than seeking an objective "place to stand", such studies of escalation emerge from and reflect the results of long term, ongoing collaborations between practitioners and researchers. Consistent with action research's best practice, what is identified as both "problem" and "solution" can be reformulated iteratively over the lifetime of the project. So far we have mainly studied the surface of what constitutes local practice in escalating situations. The next crucial question to answer is why the interpreted local practice is seen as a logical part of institutional practice. Questions to ask here include: Why is it perfectly logical for a senior midwife to call a much more junior physician to take over the responsibility of a non-normal situation? How does a crisis "deform" traditional, habitual, even historically determined local clinical practice, especially the ones practiced across and between clinical specialities? Clinical practice of course is local, as are all responses to emergency situations. But how does local practice change when crises occurs and escalates? It is this escalation, this shift in tempo, that sets off a crisis from normal events and this qualitative change is central for us to understand if we are to build an "anatomy of escalation" in much the same way as Plumb et al. argued for an "anatomy of patient safety" in a previous issue of this journal [63].

The more pragmatic implication of the research concerns the ways in which healthcare practitioners are now prepared for the complexities of escalating situations. Rather than preparing for crisis management by fostering "more appropriate" behaviour of individuals, the focus on escalation turns towards problems of interaction, coordination and enhancing organisational diversity [64]. In times when "production efficiency" is the most celebrated aim of the healthcare system (at least in the Scandinavian country that we have studied) just providing the possibility for 
midwives, obstetricians, anaesthesiology nurses, operation ward nurses, anaesthesiologists, paediatric nurses and paediatricians to meet and problematise issues concerning their interactions in the times that they become tightly coupled and highly interdependent, could be seen as a success. We hope to bring readers of this journal reports soon about the outcomes of such processes.

If we better understand not only how local practices are exploited in crisis but also how local practice can deform under stress, this could lead to innovation in both knowledge and practice. Using terms like escalation, event chains and choreography could help us strengthened the analysis of clinical acts and competence. This can help us turn our attention, as all good models should, to important elements in an important class of social phenomenon, crises and emergencies, that we so far have overlooked.

\section{End notes}

${ }^{a}$ The notion of coupling is here used much in the same way as in Snook's analysis of a Friendly-fire accident over northern Iraq in 1994 [15]. Analysing how diverse actors adapted locally under years of selfgovernance what was locally rational behaviour in "normal" circumstances made the system fail in the "nonnormal" situation when the actors became tightly coupled. The locally rational behaviour of all actors involved, according to Snook, made two US F15 pilots shoot down the own Army's helicopters once the actors became tightly coupled and highly interdependent.

${ }^{b}$ In the Scandinavian country that we studied, midwives are specialist nurses with a significant medical authority. Unless the situation deviates from what is considered "normal" pregnancies as well as labours are facilitated by specialist nurses (midwives) without involvement of physicians.

${ }^{c}$ An event chain has much in common with genealogy "tree" but rather than trace family or inheritance, it enables us to track the "hand off" of resources, knowledge, and practice, within and between, that occur between different healthcare domains routinely and in emergencies. The term choreography refers to issues of tempo, pace and timing as well as the differential reliance, informed both historically and by the moment, to healthcare/clinical resources.

\section{Competing interests}

The authors declare that they have no competing interests.

\section{Author details}

'Lund University Centre for Risk Assessment and Management, PO-Box 118SE-22100, Lund, Sweden. ${ }^{2}$ Griffith University, School of Humanities, Brisbane, Australia. ${ }^{3}$ Ball State University, Department of Anthropology, Muncie, IN, USA. ${ }^{4}$ Karolinska Institute, Department of Women and Child Health, Stockholm, Sweden.

\section{Authors' contributions}

$\mathrm{JB}, \mathrm{PhD}$, has the social processes of escalation in health care settings as his main focus. He had the main responsibility of preparing the manuscript. IA-W, $\mathrm{MD}, \mathrm{PhD}$, being an obstetrician, provided the medical expertise in the study. She is also involved in data collection activities together with JB. JMN, PhD, and $\mathrm{SD}, \mathrm{PhD}$, both assisted in conducting the analysis. All the authors provided important contributions for the preparation of the manuscript. All authors read and approved the final manuscript.

\section{Received: 15 July 2011 Accepted: 15 June 2012}

Published: 15 June 2012

\section{References}

1. Runciman WB, Merry AF: Crises in clinical care: an approach to management. Qual Saf Health Care 2005, 14:156-163.

2. Heath R: Dealing with the complete crisis-the crisis management shell structure. Saf Sci 1998, 30:139-150.

3. Hamman WR: The complexity of team training: what we have learned from aviation and its applications to medicine. BMJ Qual Saf 2004, 13:i72

4. Künzle B, Zala-Mezö E, Wacker J, Kolbe M, Spahn DR, Grote G: Leadership in anaesthesia teams: the most effective leadership is shared. Qual Saf Health Care 2010, 19:e46.

5. Wright MC, Taekman JM, Endsley MR: Objective measures of situation awareness in a simulated medical environment. BMJ Qual Saf 2004, 13:i65.

6. Yule S, Flin R, Maran N, Youngson G, Mitchell A, Rowley D, Paterson-Brown S: Debriefing surgeons on non-technical skills (NOTSS). Cognit Tech Work 2008, 10:265-274.

7. Yule S, Flin R, Paterson-Brown S, Maran N: Non-technical skills for surgeons in the operating room: A review of the literature. Surgery 2006, 139:140-149.

8. Paige J, Kozmenko V, Morgan B, Howell DS, Chauvin S, Hilton C, Cohn I, O'Leary JP: From the flight deck to the operating room: an initial pilot study of the feasibility and potential impact of true interdisciplinary team training using high-fidelity simulation. J Surg EduC 2007, 64:369-377.

9. Blum R, Md MSE, Raemer D, Carroll J, Dufresne R, Cooper J: A Method for Measuring the Effectiveness of Simulation-Based Team Training for Improving Communication Skills. Anesth Analg 2005, 100:1375-1380.

10. Flin R, Maran N: Identifying and training non-technical skills for teams in acute medicine. BMJ Qual Saf Health Care 2004, 13:180.

11. Salas E, DiazGranados D, Klein C, Burke CS, Stagl Kevin C, Goodwin Gerald F, Halpin Stanley M: Does Team Training Improve Team Performance? A Meta-Analysis. Hum Factors 2008, 50:903-933.

12. Manser T: Teamwork and patient safety in dynamic domains of healthcare: a review of the literature. Acta Anaesthesio/ Scand 2009, 53:143-151.

13. Rabøl LI, Østergaard D, Mogensen T: Outcomes of classroom-based team training interventions for multiprofessional hospital staff. A systematic review. BMJ Qual Saf Health Care 2010, 19:e27.

14. Masiello I: Why simulation-based team training has not been used effectively and what can be done about it. Adv Health Sci Educ Theory Pract 2011. doi:10.1007/s10459-011-9281-8.

15. Snook SA: Friendly fire, the accidental shootdown of U.S Black hawks over northern Iraq. Princeton: Princeton University Press; 2000.

16. Feltovich PJ, Ford KM, Hoffman RR: Expertise in context: human and machine. Menlo Park, Calif: AAAI Press; Cambridge, Mass.: MIT Press; 1997.

17. Dekker SWA: Ten questions about human error: a new view of human factors and system safety/Sidney WA. Dekker. Mahwah, N.J: Lawrence Erlbaum Associates; 2005

18. Amalberti R, Vincent C, Auroy $Y$, de Saint Maurice G: Violations and migrations in health care: A framework for understanding and management. BMJ Qual Saf Health Care 2006 2006, 15(Suppl 1):i66-i71.

19. Dekker SWA, Bergström J, Amer-Wåhlin I, Cilliers P: Complicated, complex, and compliant: Best practice in obstetrics. Cognit Tech Work 2011. doi:10.1007/s10111-011-0211-6.

20. Dekker SWA: Drift into failure: from hunting broken components to understanding complex systems. Farnham; Burlington, VT: Ashgate Pub; 2011.

21. Dekker SWA: Failure to adapt or adaptations that fail: contrasting models on procedures and safety. Appl Ergon 2003, 34:233-238.

22. Holmes D, Murray SJ, Perron A, McCabe J: Nursing Best Practice Guidelines: reflecting on the obscene rise of the void. J Nurs Manag 2008, 16:394-403 
23. Woods DD, Patterson ES, Cook Rl: Behind human error: taming complexity to improve patient safety. In Handbook of Human Factors and Ergonomics in Health Care and Patient Safety. London: Lawrence Erlbaum; 2007:459-476.

24. Cook Rl, Render M, Woods DD: Gaps in the continuity of care and progress on patient safety. BMJ 2000, 320:791-794.

25. Nemeth CP, Cook Rl, Woods DD: The messy details: insights from the study of technical work in healthcare. IEEE Trans Syst Man Cyber A Syst Hum 2004, 34:689-692.

26. Cook Rl, Woods DD: Adapting to new technology in the operating room. Hum Factors 1996, 38:593-613.

27. Dinka $D$, Nyce JM, Timpka T: GammaKnife surgery: safety and the identity of users. Technol Health Care 2005, 13:485-495.

28. Dinka D, Nyce JM, Timpka T, Holmberg K: Adding Value with 3D Visualization and Haptic Forces to Radiosurgery-A Small Theory-Based, Quasi-Experimental Study. J med syst 2006, 30:293-301.

29. Dinka D, Nyce JM, Timpka T: The need for transparency and rationale in automated systems. Interacting with Computers 2006, 18:1070-1083.

30. Miller A, Xiao Y: Multi-level strategies to achieve resilience for an organisation operating at capacity: a case study at a trauma centre. Cognit Tech Work 2007, 9:51-66.

31. Xiao Y, Kiesler S, Mackenzie CF, Kobayashi M, Plasters C, Seagull FJ, Fussell S: Negotiation and conflict in large scale collaboration: a preliminary field study. Cognit Tech Work 2007, 9:171-176.

32. Bergström J, Dahlström N, Henriqson E, Dekker S: Team Coordination in Escalating Situations: An Empirical Study Using Mid-Fidelity Simulation. J Contingencies Crisis Manag 2010, 18:220-230.

33. Woods D, Patterson E: How unexpected events produce an escalation of cognitive and coordinative demands. In Stress workload and fatigue. Edited by Hancock P, Desmond P. Hillsdale NJ: Lawrence Erlbaum; 2001.

34. Healy S: Epistemological pluralism and the [] politics of choice'. Futures 2003, 35:689-701.

35. Mintzberg $\mathrm{H}$ : The structuring of organizations: A synthesis of the research London: Prentice-Hall; 1979.

36. Feld MD: Information and authority: The structure of military organization. Am Sociol Rev 1959, 24:15-22.

37. Rochlin Gl, La Porte TR, Roberts KH: The self-designing high-reliability organization: Aircraft carrier flight operations at sea. Naval War College Rev 1987, 40:76-90.

38. Weick KE, Sutcliffe KM: Managing the Unexpected, Resilient Performance in an Age of Uncertainty. San Francisco: Jossey-Bass; 2007.

39. Rochlin Gl: Defining" high reliability" organizations in practice: A taxonomic prologue. In New challenges to understanding organizations. Edited by Roberts KH. New York: Macmillan; 1993:11-32.

40. Sagan SD: The limits of safety: organizations, accidents and nuclear weapons. Princeton: Princeton University Press; 1993

41. Wildavsky AB: Searching for safety. New Brunswick, USA: Transaction Books; 1988.

42. LaPorte TR, Consolini PM: Working in practice but not in theory: theoretical challenges of" high-reliability organizations". J Publ Admin Res Theor: J-PART 1991, 1:19-48,

43. Vaughan D: Slopes, repeating negative patterns, and learning from mistake? In Organization at the Limit: Lessons from the Columbia Disaster. Edited by Starbuck WH, Farjoun M. Malden MA: Blackwell Publishing; 2005:41-59.

44. Pronovost PJ, Vohr E: Safe patients, smart hospitals: how one doctor's checklist can help us change health care from the inside out. New York: Plume; 2011.

45. Berry NS: Kaqchikel midwives, home births, and emergency obstetric referrals in Guatemala: Contextualizing the choice to stay at home. Soc Sci Med 2006, 62:1958-1969.

46. Riewpaiboon W, Chuengsatiansup K, Gilson L, Tangcharoensathien V: Private obstetric practice in a public hospital: mythical trust in obstetric care. Soc Sci Med 2005, 61:1408-1417.

47. Wagner M: Fish can't see water: The need to humanize birth. Int J Gynaecol Obstet Office Organ Int Fed Gynaecol Obstet 2001, 75(Suppl 1):25-37.

48. Farrington-Darby T, Wilson JR: The nature of expertise: a review. App/ Ergon 2006, 37:17-32.

49. Bosk CL: Forgive and remember. London: University of Chicago Press; 2003

50. Helmreich RL, Schaefer HG: Team performance in the operating room. In Human error in medicine. Edited by Bogner MS. Hillsdale, NJ: Lawrence Erlabaum Associates; 1994:225-253.

51. Lingard L, Espin S, Whyte S, Regehr G, Baker GR, Reznick R, Bohnen J, Orser B, Doran D, Grober E: Communication failures in the operating room: an observational classification of recurrent types and effects. Qual Saf Health Care 2004, 13:330.

52. Patterson ES, Cook RI, Woods DD, Render ML: Examining the complexity behind a medication error: generic patterns in communication. Syst Man Cybern A Syst Hum IEEE Transact 2004, 34:749-756.

53. Mackintosh N, Sandall J: Overcoming gendered and professional hierarchies in order to facilitate escalation of care in emergency situations: the role of standardised communication protocols. Soc Sci Med 2010, 71:1683-1686.

54. Dekker S: The criminalization of human error in aviation and healthcare: A review. Saf Sci 2011, 49:121-127.

55. Dekker SWA: Illusions of explanation: A critical essay on error classification. Int J Aviat Psychol 2003, 13:95-106.

56. Dekker S: Just Culture, Balancing Safety and Accountability. Aldershot: Ashgate Publishing Limited; 2008.

57. Dekker SW: Criminalization of medical error: who draws the line? ANZ J Surg 2007, 77:831-837.

58. Grint K: Problems, problems, problems: The social construction of 'leadership'. Hum Relat 2005, 58:1467.

59. Brown HC, Paranjothy S, Dowswell T, Thomas J: Package of care for active management in labour for reducing caesarean section rates in low-risk women. Cochrane Database Syst Rev 2008, 8(4):CD004907.

60. Foucault M, Gordon C: Power/Knowledge: Selected interviews and other writings, 1972-1977. Ed.Colin Gordon, trns. Colin Gordon et al. New York: Pantheon; 1980

61. Foucault M: The Order of Things: An Archaeology of the Human Sciences. 1966. New York: Vintage; 1973.

62. Brydon-Miller M, Greenwood D, Maguire P: Why action research? Action Res 2003, 1:9.

63. Plumb J, Travaglia J, Nugus P, Braithwaite J: Professional conceptualisation and accomplishment of patient safety in mental healthcare: an ethnographic approach. BMC Health Serv Res 2011, 11:100

64. Bergström J, Henriqson E, Dahlström N: From crew resource management to operational resilience. In Proceedings of the 4th symposium on resilience engineering, sophia antipolis, france, june 8-10 2011. Edited by Hollnagel $E_{,}$ Rigaud E, Besnard D. Paris: Presses des Mines; 2011

doi:10.1186/1472-6963-12-161

Cite this article as: Bergström et al:: The social process of escalation: a promising focus for crisis management research. BMC Health Services Research 2012 12:161.

\section{Submit your next manuscript to BioMed Central and take full advantage of:}

- Convenient online submission

- Thorough peer review

- No space constraints or color figure charges

- Immediate publication on acceptance

- Inclusion in PubMed, CAS, Scopus and Google Scholar

- Research which is freely available for redistribution 